\title{
Ultra-high energy multi-messengers at the Pierre Auger Observatory
}

\author{
Jaime Alvarez-Muñiz* \\ Depto. Física de Partículas \& Instituto Galego de Física de Altas Enerxías \\ Universidade de Santiago de Compostela, 15782 Santiago de Compostela, Spain \\ for the Pierre Auger Collaboration \\ Observatorio Pierre Auger, Av. San Martín Norte 304, 5613 Malargüe, Argentina \\ E-mail: auger_spokespersonsefnal.gov \\ Full author list at: http://www.auger.org/archive/authors_2016_09.html
}

The Pierre Auger Observatory is the largest ultra-high energy cosmic-ray detector in the world. With the Surface Detector array of the Observatory we can also search for neutral particles that point back to their sources of production. In particular, ultra-high energy neutrinos and photons, with energies around $1 \mathrm{EeV}$ and above, can be identified, and the most stringent limits at EeV energies are set to their fluxes from a large fraction of the sky. These limits provide additional information on the nature of the ultra-high energy cosmic rays which may help identifying the sources where they are accelerated up to $100 \mathrm{EeV}$.

Neutrino Oscillation Workshop

4 - 11 September, 2016

Otranto (Lecce, Italy)

\footnotetext{
* Speaker.
} 
The nature and origin of the ultra-high energy cosmic rays (UHECRs) in the EeV $\left(10^{18} \mathrm{eV}\right)$ energy range and above is a long-standing question in Astroparticle Physics. The energy spectrum of UHECRs has been measured with unprecedented precision at the Pierre Auger Observatory, the largest UHECR observatory in the world [1]. Among the features in the spectrum there is a clear suppression of the flux above $E \sim 50 \mathrm{EeV}$ [2] that is compatible with the interaction of UHECRs with the cosmic microwave background (CMB) radiation. An alternative explanation is a scenario where the limiting energy of the UHECR sources is being observed. The determination of the mass of the UHECRs is crucial to discriminate between these possibilities with the second scenario typically predicting primaries heavier than protons at the highest energies. Indeed, the composition measurements performed at Auger could be interpreted as an evolution from light to heavier nuclei if hadronic interaction models describe well air shower physics [3], but the situation is not yet clear. More hints on the origin of the UHECRs can be obtained from the analysis of the arrival directions of the UHECRs collected at Auger. These studies have revealed a large degree of isotropy around $\sim 50 \mathrm{EeV}$ and at a few degrees angular scales [4]. The analysis of large angular scales has provided hints of a dipolar anisotropy of $\sim 7 \%$ amplitude for $E>8 \mathrm{EeV}$ [4] whose origin is being investigated.

Being charged particles, UHECRs are deflected in the galactic and extragalactic magnetic fields and lose information about the sites where they were produced. However, if UHECRs interact within the sources and/or in propagation through the Universe then we expect photons and neutrinos pointing back to their sources, generated through neutral and charged pion production and their decay. The (non-)observation of these neutral particles can provide further hints on the dominant scenario of UHECR production. The Pierre Auger Observatory has excellent sensitivity to $\mathrm{EeV}$ photon and neutrino fluxes due to its vast collecting area and its ability to discriminate between photons or neutrinos and hadronic cosmic rays. In this contribution we summarize these capabilities and recent results on the search for neutral particles at the Pierre Auger Observatory.

\section{Searching for UHE photons and neutrinos in Auger}

The Pierre Auger Observatory is located near the town of Malargüe in Mendoza, Argentina. It consists of a surface detector array (SD) with 1660 water-Cherenkov detectors (WCD) spread over an area of $\sim 3000 \mathrm{~km}^{2}$, separated by $1.5 \mathrm{~km}$ and arranged in a triangular grid. These WCD sample the shower front at the ground level, and the signals produced by the passage of shower particles are digitised with $25 \mathrm{~ns}$ resolution. There are also 27 telescopes of the air fluorescence detector that are used to measure the longitudinal development of air showers above the surface array. A fraction of events are detected in hybrid mode with the SD and FD [1].

\subsection{Photons}

Showers induced by photons are characterised by a lower content of muons and larger average depth of maximum longitudinal development $\left(X_{\max }\right)$ than hadronic showers. The photon detection efficiency of the SD enables a photon search with large event statistics at energies above $10 \mathrm{EeV}$ [5]. The reduced muon content of photon showers with respect to data (dominated by hadronic background) brings as a consequence that photon showers typically have smaller signals than data at large distances from the shower axis. Also the spread in the arrival time of secondary particles in 
individual stations in photon showers increases with respect to hadronic showers due to the larger contribution of the electromagnetic component, and to the larger $X_{\max }$. The analysis based on these two ideas constrains the integral photon flux above $10 \mathrm{EeV}$ (arrows marked as "SD2015" in Fig. 1).

A second analysis is based on the detection of air-showers in hybrid mode [6]. The identification of photon-induced air showers relied on the measurement of $X_{\max }$, while SD information was used to constrain the geometry of the showers, but not directly in the photon identification. Upper limits at the level of a few percent of the total UHECR flux were placed on the integral photon fraction above $2 \mathrm{EeV}$ (arrows marked as "Hy 2011" in Fig. 1). A novel analysis combining $X_{\max }$ and the signal at the ground was recently performed [7]. The use of combined SD and FD observables for photon identification in data from January 2015 up to December 2013 improved background rejection and the use of a less stringent data selection allowed to achieve sensitivities to photon fractions down to $0.1 \%$ and to $E=1 \mathrm{EeV}$ (arrows marked as "Hy 2016" in Fig. 1). Auger limits put severe constrains on top-down models with UHECRs originating from the decay of supermassive particles. Also UHE photon production in interactions of protons with the CMB (cosmogenic photons) starts to be challenged [5,7]. The production of UHE photons at astrophysical sources has also been tested performing blind searches for excesses of hybrid photon-like events over the sky exposed to the Auger Observatory [8]. These analyses considered events with $E<3 \mathrm{EeV}$. The non-observation of photon candidates implies that either the extra-galactic sources of UHECRs are farther than $\sim 5 \mathrm{Mpc}$ out of the distance reach of EeV photons, or they are Galactic but are transient or beamed, or the optical depth for UHECR interactions in the sources is small.

\subsection{Neutrinos}

Neutrinos can initiate showers very deep in the atmosphere while protons and heavier nuclei interact shortly after entering it. At large zenith angles the atmosphere is thick enough so that the electromagnetic component of nucleonic-cosmic rays gets absorbed and the shower front at the ground level is dominated by muons. On the other hand, showers induced by neutrinos deep in the atmosphere have a considerable amount of electromagnetic component at the ground. With the SD we can distinguish narrow signals in time induced by inclined showers initiated high in the atmosphere, from the broad signals expected in inclined showers initiated close to the ground. No neutrino candidates were found in data collected between 1 January 2004 and 20 June 2013 after applying the neutrino selection criteria described in [9]. The 90\% C.L. single-flavor limit to the diffuse flux of UHE neutrinos with an $E^{-2}$ spectrum is: $E_{v}^{2} d N_{v} / d E_{v}<6.4 \times 10^{-9} \mathrm{GeV} \mathrm{cm}^{-2} \mathrm{~s}^{-1} \mathrm{sr}^{-1}$, see Fig. 1. Cosmogenic $v$ models that assume a pure primary proton composition injected at the sources of UHECRs and strong (FRII-type) evolution are severely disfavored by Auger data. A large range of top-down models of UHECR production are excluded at $>99 \%$ C.L.

The set of neutrino identification criteria in [9] were also applied to data collected around the UTC times of occurence of the gravitational-wave (GW) events detected by Advanced LIGO namely, GW150914, GW151226 and the candidate event LVT151012 [11]. The events were inferred to have arisen from the merger of black holes in binary systems. No neutrino candidates were found in the time windows $\pm 500 \mathrm{~s}$ around or 1 day after GW150914, GW151226 or LVT151012 [10] and we placed upper limits on the energy emitted per flavor in UHE neutrinos. The limits depend on the declination of the mergers which is poorly constrained by LIGO observations. The most restrictive limits placed by Auger, and expressed as fractions of energy in UHE neutrinos rela- 
tive to the energy radiated in GW are $~ 14 \%$ in the case of GW150914 and $\sim 44 \%$ for GW151226, constraining the production of UHECRs in mergers of binary black holes [10].

\section{Conclusion}

The lack of observation of UHE photons and neutrinos by the Pierre Auger Collaboration provides important clues on the origin and nature of the UHECRs.

\section{References}

[1] A. Aab et al., [Pierre Auger Collab.], Nucl. Instrum. Meth. A 798172 (2015).

[2] J. Abraham et al. [Pierre Auger Collaboration], Phys. Rev. Lett. 101 (2008) 061101.

[3] A. Aab et al. [Pierre Auger Collaboration], Phys. Rev. D 90 (2014) 122005.

[4] J. Aublin for the Pierre Auger Collaboration, PoS(ICRC2015) 310 (2015); I. Al-Samarai for the Auger Collab., PoS(ICRC2015) 372 (2015) [arXiv:1509.03732].

[5] C. Bleve for the Pierre Auger Collaboration, PoS(ICRC2015) 1103 (2015) [arXiv:1509.03732].

[6] J. Abraham et al. [Pierre Auger Collaboration], Astropart. Phys. 31 (2009) 399; M. Settimo for the Auger Collab., Procs. of the $32^{\text {nd }}$ Intl. Cosmic Ray Conf. 2 (2011) 55 [arXiv:1107.4805].

[7] A. Aab et al. [Pierre Auger Collaboration], [arXiv:1612.01517v1].

[8] A. Aab et al. [Pierre Auger Collaboration], Astrophys. J. 789 (2014) 160.

[9] A. Aab et al. [Pierre Auger Collab.], Phys. Rev. D 91 (2015) 092008.

[10] A. Aab et al. [Pierre Auger Collab.], [arXiv:1608.07378].

[11] B. P. Abbott et al. [LIGO Scientific Collab. and Virgo Collab.], Phys. Rev. X 6041015 (2016). 

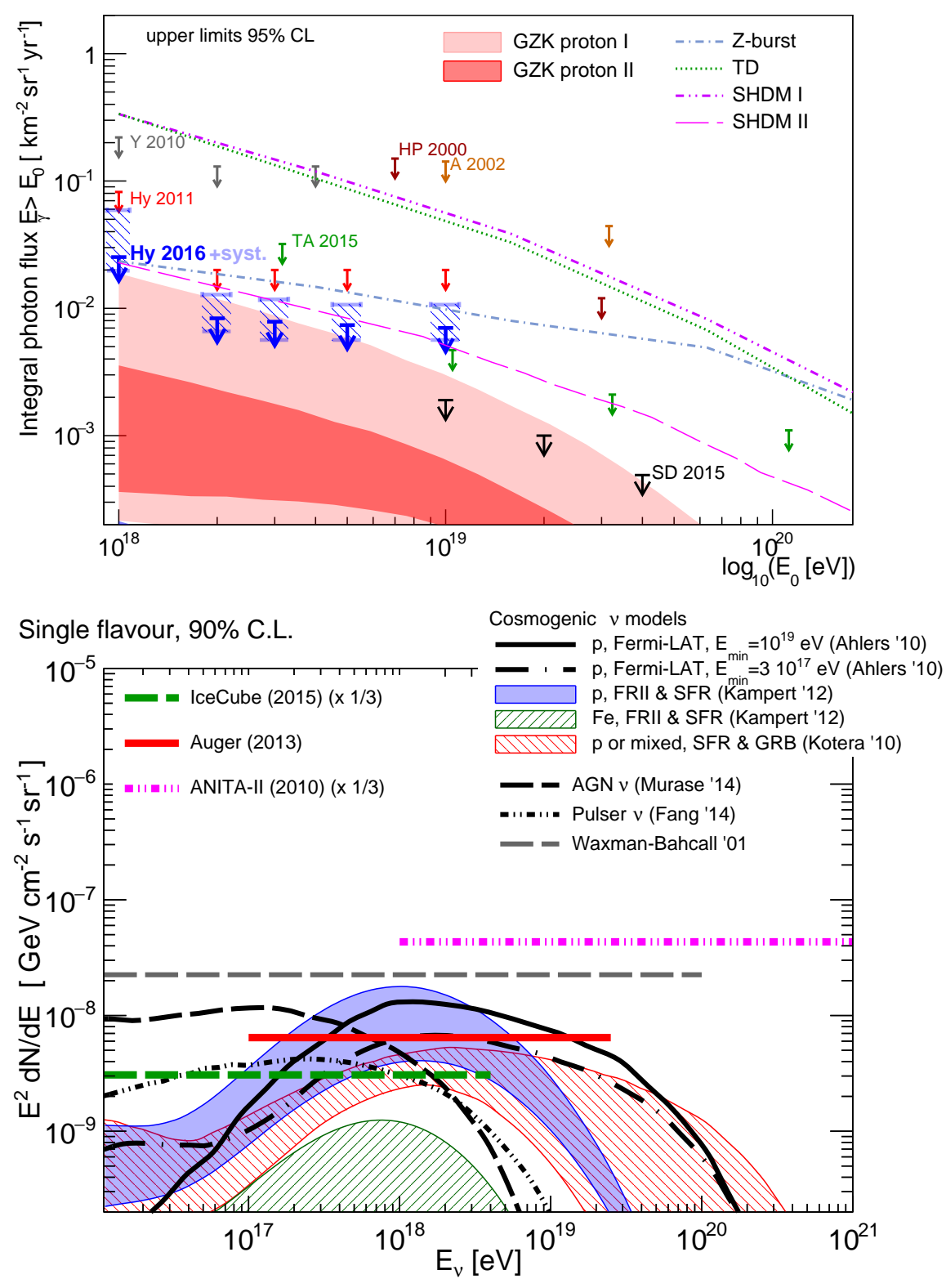

Figure 1: Top panel. Upper limits on the integral photon flux: Limits from Auger (Hy 2016 [7], SD 2015 [5] and Hy 2011 [6]), Telescope Array (TA), AGASA (A), Yakutsk (Y) and Haverah Park (HP). Shaded regions and lines: Predictions for the cosmogenic photon flux assuming pure protons at the sources and for top-down models (TD, Z-Burst, SHDM I and SHDM II) - see [7] for full references. Bottom panel: 90\% C.L. upper limit to the normalization $k$ of the diffuse flux of UHE neutrinos from Auger along with the limits from ANITAII and IceCube. We also show the predictions from several cosmogenic neutrino models, models of neutrino production at astrophysical sources as well as the Waxman-Bahcall bound. All limits and fluxes are converted to single flavor (see [9] for full references). 